\title{
RINGS OVER WHICH FLAT COVERS OF SIMPLE MODULES ARE PROJECTIVE
}

\author{
ENGIN BÜYÜKAŞIK \\ Izmir Institute of Technology \\ Department of Mathematics \\ 35430, Urla, Izmir, Turkey \\ enginbuyukasik@iyte.edu.tr \\ Received 27 May 2010 \\ Accepted 28 June 2011 \\ Communicated by J. Trlifaj
}

\begin{abstract}
Let $R$ be a ring with identity. We prove that, the flat cover of any simple right $R$-module is projective if and only if $R$ is semilocal and $J(R)$ is cotorsion if and only if $R$ is semilocal and any indecomposable flat right $R$-module with unique maximal submodule is projective.
\end{abstract}

Keywords: Flat cover; projective module; perfect ring.

Mathematics Subject Classification: 16E30, 16N80

\section{Introduction}

Throughout, $R$ is a ring with an identity element and all modules are unital right $R$ modules. Let $\mathcal{F}$ be the class of all flat right $R$-modules. Following [9], an $\mathcal{F}$-precover of an $R$-module $M$ is a homomorphism $\varphi: F \rightarrow M$ with $F \in \mathcal{F}$ such that for any $\varphi^{\prime}: F^{\prime} \rightarrow M$ with $F^{\prime} \in \mathcal{F}$, there exists a homomorphism $f: F^{\prime} \rightarrow F$ such that $\varphi^{\prime}=\varphi f$. An $\mathcal{F}$-precover $\varphi: F \rightarrow M$ is said to be an $\mathcal{F}$-cover if every endomorphism $f$ of $F$ with $\varphi f=\varphi$ is an isomorphism. For an $R$-module $M$, an epimorphism $f: P \rightarrow M$ is said to be a projective cover of $M$ if $P$ is projective and $\operatorname{Ker} f \ll P$. In [7] Bican et al. proved that all modules have flat covers. In contrast, an arbitrary module may not have a projective cover, in general. The rings over which all right modules have projective covers are exactly the right perfect rings (see, [8]). In this case, it is of interest to know when the flat cover of a given module is projective. It is well known that, a ring $R$ is right perfect if and only if flat cover of any right $R$-module is projective. The rings with the property that flat covers of finitely generated right $R$-modules are projective are characterized in $[1,2]$. In $[1,2]$, a ring $R$ is called almost-perfect ( $A$-perfect) if every flat $R$-module is $R$-projective. 
They proved that the right $A$-perfect rings are exactly those rings $R$ over which flat covers of finitely generated modules are projective.

Let $R$ be a ring and $\mathcal{S}$ be the class of all representatives of simple right $R$-modules (i.e. each element of $\mathcal{S}$ is isomorphic to $R / I$ for some maximal right ideal $I$ of $R$ ). We call $R$ right $B$-perfect if for every flat module $F$ and $S \in \mathcal{S}$, and homomorphisms $f: R \rightarrow S, h: F \rightarrow S$ there exists a homomorphism $g: F \rightarrow R$ such that $h=f g$. It is clear that any right $A$-perfect ring is right $B$-perfect.

The main objective of this paper is to introduce and give several characterizations of the rings over which flat covers of simple modules are projective. We prove that, $R$ is right $B$-perfect if and only if flat covers of simple right $R$-modules are projective if and only if $R$ is semiperfect and flat covers of simple modules are finitely generated (Theorem 2.4). We also prove that, $R$ is right $B$-perfect if and only if $R$ is semilocal and $J(R)$ is cotorsion if and only if $R$ and the maximal right ideals of $R$ are cotorsion (Theorem 2.11).

For a ring $R$ and a right $R$-module $M, J(R)$ and $J(M)$ will stand for the Jacobson radical of $R$ and the Jacobson radical of $M$, respectively.

\section{Characterizations of Right $B$-Perfect Rings}

The following lemma characterizes when the flat cover of a given module is projective. The proof is easy and standard, we include it for completeness.

Lemma 2.1. Let $\varphi: F \rightarrow M$ be a flat cover of $M$. Then, the following are equivalent.

(1) $F$ is projective.

(2) There exists an epimorphism $f: P \rightarrow M$ with $P$ projective such that the induced map $\operatorname{Hom}(F, P) \rightarrow \operatorname{Hom}(F, M)$ is surjective.

(3) There exists a flat precover $f: P \rightarrow M$ with $P$ projective.

Proof. $(1) \Rightarrow(2)$ : Take $P=F$, then the proof is clear.

$(2) \Rightarrow(3)$ : Let $G$ be a flat module and $h \in \operatorname{Hom}(G, M)$. By (2) there exist $\alpha \in \operatorname{Hom}(F, P)$ such that $\varphi=f \alpha$. On the other hand, since $\varphi: F \rightarrow M$ is a flat cover, $h=\varphi \beta$ for some $\beta \in \operatorname{Hom}(G, F)$. Hence we obtain the following diagram:

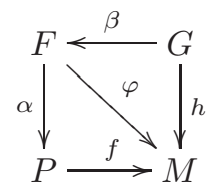

In this case, from $h=\varphi \beta$ and $\varphi=f \alpha$, we get $h=f(\alpha \beta)$ with $\alpha \beta \in \operatorname{Hom}(G, P)$. This proves (3).

$(3) \Rightarrow(1)$ : By $[9$, Theorem 1.2.7], $M$ has a flat cover that is a direct summand of $P$. Therefore $F$ is projective, because flat covers (of $M$ ) are isomorphic (see, [9, Theorem 1.2.6]). 
In [1, Theorem 3.7], it is shown that a $\operatorname{ring} R$ is right $A$-perfect if and only if the flat cover of any finitely generated right $R$-module is projective. From Lemma 2.1 we see that, an arbitrary module has a projective flat cover if and only if it has a projective flat precover. Hence the following corollary is clear.

Corollary 2.2. A ring $R$ is right A-perfect if and only if any finitely generated right $R$-module has a projective flat precover.

Recall that a ring $R$ is right (or left) semiperfect if $R$ is semilocal (i.e. $R / J(R)$ is semisimple artinian) and the idempotents of $R / J(R)$ lift to $R$. A ring $R$ is semiperfect if and only if every simple right (left) $R$-module has a projective cover $[8,42.6]$. Over a semiperfect ring any finitely generated flat right $R$-module is projective. As we have mentioned, a flat cover $\varphi: F \rightarrow M$ of a module $M$ need not be a projective cover. But, in case, $F$ is projective then $\operatorname{Ker} \varphi \ll F$ and so $\varphi: F \rightarrow M$ is a projective cover (see, $[9$, Theorem 1.2.12]). We shall use these facts in the sequel. Recall that, if $R$ is a semilocal ring then $J(M)=M . J(R)$ for any right $R$-module $M$ (see, [3, Corollary 15.18]).

Lemma 2.3. Let $R$ be a semilocal ring and $X_{R}$ be a simple right $R$-module. If $\phi: F \rightarrow X$ is the flat cover of $X$, then the kernel $\operatorname{Ker} \phi=F . J(R)$. Moreover, $F$ is indecomposable.

Proof. See the proof of [6, Corollary 23].

Theorem 2.4. For a ring $R$ the following statements are equivalent:

(1) $R$ is right $B$-perfect.

(2) Flat covers of simple modules are projective.

(3) $R$ is semiperfect and flat covers of simple modules are cyclic.

(4) $R$ is semiperfect and flat covers of simple modules are local.

(5) Flat covers of finitely generated semisimple modules are projective.

(6) $R$ is semiperfect and flat covers of finitely generated semisimple modules are finitely generated.

Proof. $(1) \Rightarrow(2)$ This is clear from the definition of $B$-perfect rings.

$(2) \Rightarrow(3)$ Let $f: F \rightarrow X$ be the flat cover of the simple right $R$-module $X$. By (2) $F$ is projective and so $\operatorname{Ker} f \ll F$. Now we have $F / \operatorname{Ker} f \simeq X$ is cyclic and Ker $f \ll F$. Hence $F$ is cyclic. On the other hand $f: F \rightarrow X$ is a projective cover of the simple $R$-module $X$. Therefore $R$ is semiperfect.

$(3) \Rightarrow(4)$ Let $f: F \rightarrow X$ be the flat cover of the simple right $R$-module $X$. We only need to prove that $F$ is local. By Lemma $2.3 \operatorname{Ker} f=F$.J where $J=J(R)$. Since $F / F . J \simeq X$ is simple and $F . J \subseteq J(F)$, we get $F . J$ is the unique maximal submodule of $F$. On the other hand $F$ is finitely generated. Therefore $F . J$ is the largest submodule of $F$, and so $F$ is a local module by [8, p. 351]. 
$(4) \Rightarrow(5)$ Finitely generated flat modules are projective over semiperfect rings. Let $M=\bigoplus_{i=1}^{n} S_{i}$ be a semisimple module and $f_{i}: F_{i} \rightarrow S_{i}$ be the flat cover of $S_{i}$ for each $i=1, \ldots, n$. Then $\bigoplus_{i=1}^{n} f_{i}: \bigoplus_{i=1}^{n} F_{i} \rightarrow \bigoplus_{i=1}^{n} S_{i}$ is a flat cover of $\bigoplus_{i=1}^{n} S_{i}$ by $\left[9\right.$, Theorem 1.2.10]. By hypothesis $F_{i}$ is projective for each $i=i, \ldots, n$, hence $\bigoplus_{i=1}^{n} F_{i}$ is projective.

$(5) \Rightarrow(6)$ Flat cover of any simple module is projective by (5), hence $R$ is semiperfect. Now, if $M$ is a finitely generated semisimple module and $\phi: F \rightarrow M$ is a flat cover, then $F$ is projective by (5). Then $\operatorname{Ker} \phi \ll F$ and this implies that $F$ is finitely generated as $F / \operatorname{Ker} \phi \simeq M$ is finitely generated.

$(6) \Rightarrow(1)$ Let $X$ be a simple module and $f: R \rightarrow X$ be an epimorphism. Let $G$ be a flat module and $g: G \rightarrow X$ be a homomorphism. If $\phi: F \rightarrow X$ is a flat cover, by (6) $F$ is finitely generated. Then $F$ is projective as $R$ is semiperfect. Therefore there is a homomorphism $h: F \rightarrow R$ such that $f=\phi h$. Then by Lemma 2.1 there is a homomorphism $t: G \rightarrow R$ such that $g=f t$. Hence $R$ is $B$-perfect.

As we have mentioned, the class of $A$-perfect rings is contained in the class of $B$-perfect rings. An example of a $B$-perfect ring which is not $A$-perfect is constructed in [1] as follows.

Example 2.5 ([1, Examples 2.17, 2.22]). Let $K$ be a field and $S=K\left[y_{1}, y_{2}, \ldots\right]$ be the polynomial ring in indeterminates $y_{1}, y_{2}, \ldots$ over $K$. Let $L$ be the ideal of $S$ generated by $\left\{y_{i} y_{j} \mid i, j=1,2, \ldots\right\}$. Consider the ring $R=S / L$. Then the ring $R[[x]]$ is not $A$-perfect by [1, Example 2.17]. On the other hand, flat cover of any simple $R[[x]]$-module is projective by $[1$, Example 2.22]. Hence $R[[x]]$ is a $B$-perfect ring by Theorem $2.4(1) \Leftrightarrow(2)$.

Theorem 2.6. Let $R$ be any ring. Then $R$ is right $B$-perfect if and only if $R$ is semilocal and any indecomposable flat right $R$-module with a unique maximal submodule is projective.

Proof. Necessity: Suppose $R$ is right $B$-perfect. Then $R$ is semiperfect by Theorem 2.4. Let $G$ be an indecomposable flat module with a unique maximal submodule $K$. Let $h: F \rightarrow G / K$ be the flat cover of the simple module $G / K$. Then $F$ is projective by Theorem 2.4, and so Ker $h \ll F$. If $\pi: G \rightarrow G / K$ is an epimorphism, then, by definition of flat cover, there exists $g: G \rightarrow F$ such that the following diagram is commutative.

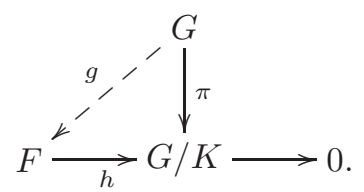

From the proof of [9, Theorem 1.2.12], $g$ is an epimorphism, and so the map $g: G \rightarrow$ $F$ splits. But $G$ is indecomposable, so that $g$ must be an isomorphism. Hence $G$ is projective. 
Sufficiency: Let $X_{R}$ be a simple module and $f: F \rightarrow X$ be the flat cover of $X$. Then $\operatorname{Ker} f=F$.J is the unique maximal submodule of $F$. Moreover $F$ is indecomposable by [5, Theorem 15]. So that $F$ is projective by the hypothesis. Hence $R$ is right $B$-perfect by Theorem 2.4 .

Definition 2.7. A right $R$-module $C$ is said to be cotorsion if $\operatorname{Ext}_{R}^{1}(F, K)=0$ for any flat right $R$-module $F$.

Lemma 2.8 ([9, Lemma 2.1.1]). Let $f: F \rightarrow M$ be a flat cover of the $R$-module $M$ and $K=\operatorname{Ker}(f)$. Then for any flat $R$-module $G, \operatorname{Ext}_{R}^{1}(G, K)=0$, i.e. $K$ is cotorsion.

Lemma 2.9 ([9, Proposition 3.3.3]). Let $I$ be an ideal of $R$ with $I \neq R$. If $C$ is cotorsion as an $R / I$-module, then it is cotorsion as an $R$-module.

The following lemma is an easy consequence of Lemma 2.9.

Lemma 2.10. Let $R$ be a semilocal ring. Then any semisimple $R$-module is cotorsion.

Proof. Suppose $M$ is a semisimple module. Then $M$ is an $R / J(R)$-module as $M . J(R)=0$. Since $R$ is semilocal, $R / J(R)$ is a semisimple ring. So that every $R / J(R)$-module is cotorsion. In particular $M$ is a cotorsion $R / J(R)$-module. Therefore $M$ is a cotorsion $R$-module by Lemma 2.9 .

It is well known that, the right perfect rings are exactly those rings $R$ over which every flat right $R$-module is projective. This implies that, $R$ is right perfect if and only if every right $R$-module is cotorsion. In [1, Theorem 2.13], the authors prove that a ring $R$ is right $A$-perfect if and only if any right ideal of $R$ is cotorsion.

We have the following corresponding result for right $B$-perfect rings.

Theorem 2.11. For a ring $R$ the following statements are equivalent:

(1) $R$ is right $B$-perfect.

(2) every right ideal of $R$ containing $J(R)$ is cotorsion.

(3) $R$ and the maximal right ideals of $R$ are cotorsion.

(4) $R$ is semilocal and $J(R)$ is cotorsion.

Proof. (1) $\Rightarrow(2)$ By Theorem 2.4, $R$ is semiperfect and flat covers of finitely generated semisimple modules are projective. Therefore flat covers and projective covers of finitely generated semisimple modules coincide. Since $J(R) \ll R$, the usual map $\pi: R \rightarrow R / J(R)$ is a projective (flat) cover. Then $\operatorname{Ker} \pi=J(R)$ is cotorsion by Lemma 2.8. Let $I$ be a right ideal containing $J(R)$. Since $R$ is semilocal and $I / J(R)$ is semisimple and finitely generated, $I / J(R)$ is a cotorsion $R$-module by Lemma 2.10. Since cotorsion modules are closed under extension and $J(R), I / J(R)$ are cotorsion, $I$ is cotorsion. 
$(2) \Rightarrow(3)$ clear.

$(3) \Rightarrow(1)$ Let $F$ be a flat module and $I_{R}$ be a maximal right ideal of $R$. By hypothesis $\operatorname{Ext}_{R}^{1}(F, I)=0$. Therefore by applying the Hom functor to the exact sequence

$$
0 \rightarrow I \rightarrow R \rightarrow R / I \rightarrow 0
$$

we get the epimorphism

$$
\operatorname{Hom}(F, R) \rightarrow \operatorname{Hom}(F, R / I) \rightarrow 0
$$

This implies that $R$ is $B$-perfect.

$(4) \Rightarrow(2)$ Let $I$ be a right ideal containing $J(R)$. Then Since $R$ is semilocal, $R / J(R)$ is semisimple, and so $I / J(R)$ is finitely generated and semisimple. Then $I / J(R)$ is a cotorsion $R$-module by Lemma 2.10. By hypothesis $J(R)$ is also cotorsion. Hence $I$ is cotorsion, because cotorsion modules are closed under extension.

$(1) \Rightarrow(4)$ By Theorem 2.4, $R$ is semiperfect, and so it is semilocal. By repeating the arguments in the proof of $(1) \Rightarrow(2)$ we obtain that $J(R)$ is cotorsion.

Example 2.12. Let $\mathbb{Z}$ be the ring of integers and $p$ be a prime integer. Consider the ring $R=\left\{\frac{a}{b} \mid a b \in \mathbb{Z},(b, p)=1\right\}$. Then $R$ is a local ring with the unique maximal ideal $p R$. The flat cover $F$ of $R / p R$ is isomorphic to the set of $p$-adic integers (see, $[9$, Theorem 1.3.8] and the example after [9, Theorem 1.3.8]). It is known that $F$ is not a projective $R$-module. Hence $R$ is not $B$-perfect. On the other hand, since $R$ is a local ring it is semiperfect.

Actually, we have the following for local rings.

Proposition 2.13. Let $R$ be a local ring. Then $R$ is $B$-perfect if and only if the usual epimorphism $\pi: R \rightarrow R / J(R)$ is a flat cover of $R / J(R)$.

Proof. Suppose $R$ is $B$-perfect. Then $J(R)$ is cotorsion by Theorem 2.11 . So that $\pi: R \rightarrow R / J(R)$ is a flat precover by [9, Proposition 2.1.3]. Let $F \rightarrow R / J(R)$ be a flat cover of $R / J(R)$. Then $F$ is a direct summand of $R$ by [9, Theorem 1.2.7]. But $R$ is indecomposable, so that $R \simeq F$. Hence $\pi: R \rightarrow R / J(R)$ is a flat cover of $R / J(R)$.

Conversely, suppose $\pi: R \rightarrow R / J(R)$ is flat cover. Then $\operatorname{Ker} \pi=J(R)$ is cotorsion, hence $R$ is $B$-perfect by Theorem 2.11 .

\section{Acknowledgment}

The author is grateful to the referee for the valuable suggestions and comments which improved the presentation of the paper. 


\section{References}

[1] B. Amini, A. Amini and M. Ershad, Almost-perfect rings and modules, Commun. Algebra 37 (2009) 4227-4240.

[2] A. Amini, M. Ershad and H. Sharif, Rings over which flat covers of finitely generated modules are projective, Commun. Algebra 36 (2008) 2862-2871.

[3] F. W. Anderson and K. R. Fuller, Rings and Categories of Modules (Springer, New York, 1992).

[4] P. A. G. Asensio and I. Herzog, Left cotorsion rings, Bull. London Math. Soc. 36 (2004) 303-309.

[5] P. A. G. Asensio and I. Herzog, Sigma-cotorsion rings, Adv. Math. 191 (2005) 11-28.

[6] P. A. G. Asensio and I. Herzog, Indecomposable flat cotorsion modules, J. Lond. Math. Soc. 76(2) (2007) 797-811.

[7] L. Bican, R. El Bashir and E. Enochs, All modules have flat covers, Bull. London Math. Soc. 33 (2001) 385-390.

[8] R. Wisbauer, Foundations of Modules and Rings (Gordon and Breach, 1991).

[9] J. Xu, Flat Covers of Modules (Springer-Verlag, Berlin, 1996). 